\title{
Detection of aberrant DNA methylation in unique Prader - Willi syndrome patients and its diagnostic implications
}

\author{
Karin Buiting+, Bärbel Dittrich+, Wendy P.Robinson', Miriam Guitart², Dvorah Abeliovich ${ }^{3}$, Israela Lerer ${ }^{3}$ and \\ Bernhard Horsthemke* \\ Institut für Humangenetik, Universitätsklinikum Essen, Hufelandstrasse 55, D-45122 Essen, Germany, 'Institut für Medizinische Genetik, Zürich, \\ Switzerland, 2Hospital de Sabadell, Sabadell, Spain and ${ }^{3}$ Department of Human Genetics, Hadassah University Hospital, Israel
}

Received January 20, 1994; Revised and Accepted April 6, 1994

\begin{abstract}
Most patients with Prader-Willi syndrome have a deletion of $15 q 11-13$ or maternal uniparental disomy for chromosome 15. The shortest region of deletion overlap is presently defined by the gene for the small nuclear ribonucleoprotein $N$ (SNRPN). We have investigated the integrity of SNRPN as well as the methylation status of D15S63 (PW71) in two patients with apparently normal chromosomes 15 of biparental origin. SNRPN is normal in one patient and deleted in the other one. Both patients are intact at the D15S63 locus, but have an abnormal methylation pattern. These results suggest that a DNA sequence close to SNRPN determines the methylation status of $\mathrm{D15S} \mathrm{3}$ and that the methylation test does not only detect the common deletions and uniparental disomy, but other rare lesions as well.
\end{abstract}

\section{INTRODUCTION}

Prader - Willi syndrome (PWS) and Angelman syndrome (AS) are distinct neurogenetic diseases. Approximately $70 \%$ of patients with PWS have a paternally derived deletion of $15 q 11-13$. Thirty per cent of patients lack a paternal chromosome 15 and have two maternal copies (uniparental disomy, UPD). These findings suggest that the gene(s) affected in PWS are expressed from the paternal chromsome 15 only. Reciprocal findings in AS indicate that the AS gene(s) are expressed from the maternal chromosome only (for review see reference 1 ). The mechanisms underlying parent-of-origin specific gene expression (imprinting) are unknown, but DNA methylation may play a major role in this process $(2-6)$.

Deletions in PWS and AS typically affect a region of $4-5 \mathrm{Mb}$, which includes the loci D15S9, D15S11, D15S13, D15S63, SNRPN, D15S10, D15S113, GABRB3, D15S97, GABRA5, D15S78 and D15S12 (7). Recently, we have identified a pair of PWS sibs (family S) who have a deletion of less than $300 \mathrm{~kb}$ (8). The deletion encompasses the gene for the small nuclear ribonucleoprotein N (SNRPN), which is active on the paternal chromosome 15 only (6), but none of the other marker loci in the region. Interestingly, these patients and another pair of PWS sibs (family 0 ), who have apparently normal chromosomes of biparental inheritance, have an aberrant DNA methylation pattern at the D15S63 (PW71) locus (8), which maps $130 \mathrm{~kb}$ proximal to SNRPN (10). Modification of the methylation pattern at this locus and at the D15S9 (ML34) locus was observed in some AS patients also $(5,8)$. Here we have investigated the integrity of SNRPN and the methylation status of D15S63 and D15S9 in two other PWS patients who by standard microsatellite analysis were found to have apparently normal chromosomes of biparental origin.

\section{RESULTS AND DISCUSSION}

Two patients with typical PWS were studied with microsatellites from 15q11-13 (Table 1). Patient $S 12$ is heterozygous at the D15S11, D15S113 and D15S97 loci and, therefore, does not have a typical deletion. Uniparental disomy was excluded by the observation of maternal and paternal alleles at D15S113 and D15S97. The presence of biparental alleles at four loci in patient 14-3 rules out a typical deletion and uniparental disomy in this patient also.

The integrity of the SNRPN gene was tested by quantitative Southern blot analysis of $B g I I I+C f o I$ digested DNA. As shown in Fig. la, the probe $S m N 1$ identifies a $7.5 \mathrm{~kb}$ band from the SNRPNP1 pseudogene on chromosome 6 and a $5.8 \mathrm{~kb}$ band from the SNRPN locus on chromosome $15(9,10$, and unpublished results). Patient $\mathrm{S} 12$ has a $5.8 \mathrm{~kb}$ band of normal intensity, whereas $14-3$ has a $5.8 \mathrm{~kb}$ band of reduced intensity. Similar results were obtained in one other independent experiment (not shown). This indicates that patient S12 is intact for SNRPN and that patient $14-3$ is deleted.

Note that the faint $5.0 \mathrm{~kb}$ band from the SNRPN locus is missing in the AS control (Fig. 1a). Similar results were obtained in three other AS deletion patients and two AS UPD patients (not shown). The difference appears to be due to parent-of-origin specific partial methylation of a $C f o I$ site within the SNRPN gene (6) and may be employed for diagnostic testing of patients suspected of having AS. It cannot be used for diagnosing PWS, because the PWS pattern is indistinguishable from the normal pattern.

Next we determined the integrity and the methylation status of D15S63. Quantitative Southern blot analysis of HindIII digested DNA with PW71 revealed a $6.6 \mathrm{~kb}$ band of normal intensity in both patients (Fig. 1b). The methylation status was determined by hybridization of a HindIII + HpaII blot and a

*To whom correspondence should be addressed

${ }^{+}$The first two authors contributed equally to this work and are listed alphabetically 
Table 1. Genotypes

\begin{tabular}{llllll}
\hline Locus & F & M & S12 & M & $14-3$ \\
\hline D15S11 & 22 & 12 & 12 & 33 & 23 \\
D15S113 & 22 & 13 & 23 & 15 & 16 \\
GABRB3 & 12 & 11 & 11 & 25 & 12 \\
D15S97 & 11 & 23 & 13 & 15 & 16 \\
\hline
\end{tabular}

F, father; $M$, mother.

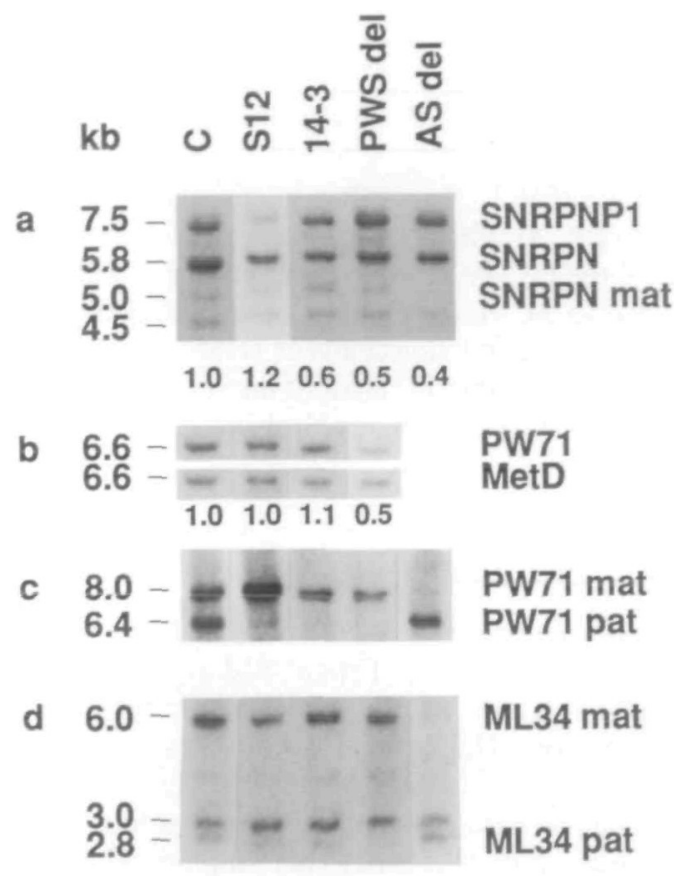

Figure 1. Southern blot analysis of patients S12, 14-3, a PWS deletion patient (PWS del), an AS deletion patient (AS del) and a normal control. mat, maternal; pat, paternal. (a) DNA from peripheral blood was digested with $B g l I I+C f o l$ and probed with $S m N 1$ (SNRPN). Signal intensities were determined with a Shimadzu Densitometer $\mathbf{C} 9000$. The normalized hybridization ratios SNRPN/SNRPNPI are given underneath each lane. Patient $S 12$ has a $5.8 \mathrm{~kb}$ SNRPN band of normal intensity, whereas patients 14-3, AS del and PWS del have a $5.8 \mathrm{~kb}$ SNRPN band of reduced intensity. The AS patient lacks the 5.0 $\mathrm{kb}$ SNRPN band. The identity of the $4.5 \mathrm{~kb}$ band is unknown. (b) DNA samples were digested with HindIII and probed with PW71B (4). After stripping, the blots were rehybridized with a probe for METD, which maps to chromosome 7. The normalized hybridization ratios PW71/METD are given underneath each lane. Patients S12 and 14-3 have a PW71 band of normal intensity, whereas PWS del has a PW71 band of reduced intensity. (c) DNA samples were digested with $B g$ III $+C f \circ \mathrm{I}$ and probed with PW71B (4). The $8.0 \mathrm{~kb}$ band and the $6.4 \mathrm{~kb}$ band represent the maternal and the paternal methylation imprint, respectively. Patients S12, 14-3 and PWS del lack the $6.4 \mathrm{~kb}$ band. Patient AS del lacks the $8.0 \mathrm{~kb}$ band. Lane S12 is slightly overloaded. (d) DNA samples were digested with HindIII + Hpall and hybridized with ML34 (2). The $6.0 \mathrm{~kb}$ band and the $2.8 \mathrm{~kb}$ band represent the maternal and the paternal methylation imprint, respectively. Patients S12 and 14-3 appear to have a normal pattern, although we cannot rule out slight quantitative differences. Patient AS del lacks the $6.0 \mathrm{~kb}$ band, whereas patient PWS del lacks the $2.8 \mathrm{~kb}$ band.

$B g I \mathrm{II}+C f o \mathrm{I}$ blot. Both patients lack the $4.7 \mathrm{~kb}$ Hind $\mathrm{dII}+\mathrm{HpaII}$ band (not shown) and the $6.4 \mathrm{~kb} \mathrm{BgIII}+C f o I$ band (Fig. 1c). These results indicate that in the two patients the HpaIl site and the CfoI site which normally are methylated on the maternal chromosome only (4) are methylated on the maternal and the paternal chromosome 15.
It is unclear why the SNRPN deletion in patient 14-3 and in the PWS siblings described by Reis et al. (8) modified the methylation imprint on the paternal chromosome. It is possible that the deletion includes a regulatory sequence which directly or indirectly determines the methylation status of the D15S63 locus. An as yet unidentified deletion or mutation of this sequence may be the cause of aberrant DNA methylation in patient $\mathrm{S} 12$ and in the non-deletion PWS siblings described by Reis $e t a l$. (8). It is tempting to speculate that this sequence controls the imprinting process in $15 q 11-q 13$.

Methylation of D15S9 (ML34) was tested with HindIII + HpaII blots. As shown in Fig. 1d, apparently normal patterns were observed in both patients. Similar findings were made in a PWS patient, who has a large deletion (B.Horsthemke et al., unpublished), as well as in the PWS siblings deleted for SNRPN only (family $\mathrm{S} ; 8$ ). Although it is possible that there are slight quantitative differences in the methylation patterns, the results suggest that ML34 methylation is less reliable for diagnostic testing.

The patients described by Reis et al. (8) and in this report demonstrate that microsatellite analysis based on the commonly used markers in 15q11-13 fails to detect some patients with PWS. This is in contrast to the PW71 methylation test, which not only detects the typical deletions and uniparental disomy (3), but other lesions also. In fact, we are not aware of any typical PWS patient with normal D15S63 methylation.

Although the methylation test is the only test that detects both deletion and non-deletion PWS, it does not distinguish between the two, unless the relative intensity of the hybridization signals is determined. This can easily be done by rehybridization of $B g / \mathrm{II}$ $+C f o$ I blots with a SNRPN probe, because the SNRPNP1 signal from chromosome 6 can serve as an internal standard to determine the copy number of PW71 and SNRPN.

The combined PW71/SNRPN test is the method of choice for rapid diagnostic testing of patients suspected of having PWS. Furthermore, it detects lesions which are undetectable by any other technique. However, the test does not provide any information on the nature and extent of a deletion. Neither does it distinguish between uniparental disomy or normal chromosomes of biparental origin (such as patient S12). This information can be obtained only by additional tests such as cytogenetic and microsatellite analysis. Specific knowledge about the etiology of PWS in a given patient is important for accurate estimates about the recurrence risk.

\section{MATERIALS AND METHODS}

Patients

Patient 14-3 is from Israel, male and 18 years old. Patient $\$ 12$ is from Spain, male and 5 years old. Both patients had severe hypotonia in early infancy and developed hyperphagia and obesity in early childhood. They have cryptorchidism, short stature, characteristic face and mental retardation. Their family history is unremarkable. The father of patient 14-3 is deceased and could not be srudied.

DNA analysis

DNA from peripheral blood was digested with $B g / \mathrm{II}+C f o \mathrm{I}$ and hybridized with SmNI (SNRPN; 10) and PW71B (D15S63; 4). For determining the copy number of PW71, HindIII digested DNA was hybridized with PW71B. After stripping, the blots were rehybridized with a probe for METD, which maps to chromosome 7. Relative signal intensities were determined with a Shimadzu Densitometer C 9000 . HindIII + HpaIl blots were used to determine the methylation status at the ML34 locus (2). Microsatellite analysis was performed as described before $(8,11,12)$. 


\section{ACKNOWLEDGEMENTS}

Part of this work was supported by the Deutsche Forschungsgemeinschaft. We thank S.Groß and B.Brandt for expert technical assistance and Prof. E.Passarge for continued support.

\section{REFERENCES}

1. Nicholls, R.D. (1993) Curr. Opinions Genet. Dev. 3, 445-456.

2. Driscoll, D.J., Waters, M.F., Williams, C.A., Zori, R.T., Glenn, C.G., Avidano, K.M. \& Nicholls, R.D. (1992) Genomics 13, 917-924.

3. Dittrich, B., Robinson W.P., Knoblauch, H., Buiting, K., Schmidt, K., Gillessen-Kaesbach, G. \& Horsthemke, B. (1992) Hum. Genet. 90, 313-315.

4. Dittrich, B., Buiting, K., Groß, S. \& Horsthemke B. (1993) Hum. Mol. Genet. 2, 1995-1999.

5. Glenn, C.G., Nicholls, R.D., Saitoh, S., Niikawa, N., Robinson, W.P., Schinzel, A., Horsthemke, B. \& Driscoll, D.J. (1993) Hum. Mol. Genet. 2, $1377-1382$.

6. Glenn, C.G., Porter, K.A., Jong, M.T.C., Nicholls, R.D. \& Driscoll, D.J. (1993) Hum. Mol. Genet. 2, $2001-2005$.

7. Kuwano, A., Mutirangura, A., Dittrich, B., Buiting, K., Horsthemke, B., Saitoh, S., Niikawa, N., Ledbetter, S.A., Greenberg, F., Chinault, A.C. \& Ledbetter, D. (1992) Hum. Mol. Genet. 1, 417-425.

8. Reis, A., Dittrich, B., Greger, V., Buiting, K., Lalande, M., GillessenKaesbach, G., Anvret, M. \& Horsthemke, B. (1994) Am. J. Hum. Genet., in press.

9. Öczelik, T., Leff, S., Robinson, W.P., Donlon, T., Lalande, M., Sanjines, E., Schinzel, A. \& Francke, U. (1992) Nature Genet. 2, 265-269.

10. Buiting, K., Dittrich, B., Groß, S., Greger, V., Lalande, M., Robinson, W., Mutirangura, A., Ledbetter, D. \& Horsthemke, B. (1993) Hum. Mol. Genet. 2, 1991-1994.

11. Mutirangura, A., Greenberg, F., Butler, M.G., Malcolm, S., Nicholls, R.D., Chakravarti, A. \& Ledbetter, D. (1993) Hum. Mol. Genet. 2, 143-151.

12. Beckmann, J.S., Tomfohrde, J., Barnes, R.I., Williams, M., Broux, O., Richard, I.,Weissenbach, J. \& Bowcock, A.M. (1993) Hum. Mol. Genet. 2, 2019-2030. 